\title{
CANSANCIO Y SOMNOLENCIA EN CONDUCTORES DE ÓMNIBUS INTERPROVINCIALES: ESTUDIO COMPARATIVO ENTRE FORMALIDAD E INFORMALIDAD ${ }^{\ddagger}$
}

\author{
Gustavo R. Liendo ${ }^{1,2, a}$ Carla L. Castro ${ }^{1,2, a}$, Jorge Rey de Castro ${ }^{1,2,3, b, c}$
}

\begin{abstract}
RESUMEN
Objetivos. Comparar los niveles de cansancio, somnolencia y sus repercusiones entre conductores formales e informales de ómnibus interprovinciales. Evaluar las condiciones laborales en ambos grupos de estudio. Materiales y métodos. Se realizó un estudio transversal comparativo con muestreo no probabilístico. Se incluyó 100 empresas de transporte terrestre, de las cuales 17 fueron formales según registros oficiales del Ministerio de Transportes y Comunicaciones (MTC), asimismo, los conductores se catalogaron como formales o informales. La encuesta incluía un cuestionario y la versión peruana validada de la escala de somnolencia de Epworth. Resultados. Participaron 71 conductores formales y 274 informales, todos fueron varones. De 134 conductores que pertenecían a las empresas formales de acuerdo al MTC, sólo $43(32 \%)$ pertenecen al grupo formal en base a los criterios propuestos. El $48 \%(34)$ de los conductores formales y el $43 \%$ (118) de los informales duermen menos de siete horas al día. Admitieron haberse accidentado o "casi accidentado" el 48\% (34) de los formales y 135 (49\%) informales; el horario más frecuente fue entre la 01.00 y 04.00 horas. La madrugada es el período en que ambos grupos sienten más cansancio. El $44 \%$ (30) de los conductores formales y el $54 \%$ (144) de informales realizan cinco o más turnos nocturnos por semana. Del total de los entrevistados, el 16\% (56) presentaron somnolencia. La asociación con accidentes de tránsito fue similar. Conclusiones. Los niveles de cansancio y somnolencia fueron similares entre conductores formales e informales. Aquellas empresas catalogadas como formales, presentan alto porcentaje de informalidad entre sus conductores.
\end{abstract}

Palabras clave: Carreteras; Accidentes de tránsito; Trastornos por excesiva somnolencia, Privación de sueño, Perú (fuente: DeCS BIREME).

\section{FATIGUE AND SLEEPINESS IN INTERPROVINCIAL ROAD BUS DRIVERS: COMPARATIVE STUDY BETWEEN FORMALITY AND INFORMALITY}

\begin{abstract}
Objectives. To compare the levels of fatigue, sleepiness and their consequences between formal and informal drivers of interprovincial buses. To evaluate labor conditions between both study groups. Materials and methods. A comparative cross-sectional study was performed with non-probabilistic sampling. 100 companies of land transport were included, out of which 17 were formal according to the official registries of the Ministry of Transport and Communications (MTC), the drivers were also classified as formal or informal. The survey included one questionnaire and a Peruvian validated version of the Epworth sleepiness scale. Results. 71 formal drivers and 274 informal drivers participated, all were males. Out of the 134 drivers that worked for the formal companies according to the MTC, only $43(32 \%)$ belong to the formal group based on the proposed criteria. $48 \%$ (34) of the formal drivers and $43 \%$ (118) of the informal sleep less than 7 hours a day. $48 \%(34)$ of the formal and $49 \%$ (135) of the informal admitted having had an accident or "almost" having had it, the most frequent time of the day was between 01.00 and 04.00 in the morning. The dawn is the period in which both groups feel most tired. $44 \%$ (30) of the formal drivers and $54 \%$ (144) of the informal ones perform 5 or more night shifts per week. Out of the total of interviewed, $16 \%(56)$ had sleepiness. The association with road traffic accidents was similar. Conclusions. The fatigue and sleepiness levels were similar between formal and informal drivers. Companies classified as formal, have a high percentage of informality amongst their drivers.
\end{abstract}

Key words: Roads; Accidents, Traffic; Disorders of excessive somnolence; Sleep deprivation; Peru (source: MeSH NLM).

\footnotetext{
Facultad de Medicina, Universidad Peruana Cayetano Heredia. Lima, Perú.

Grupo de Investigación en Sueño (GIS). Lima, Perú.

Centro de Trastornos Respiratorios del Sueño (CENTRES), Clínica Anglo Americana. Lima, Perú.

Médico Cirujano; ${ }^{\mathrm{b}}$ Médico neumólogo; ${ }^{\mathrm{c}}$ Magíster en Medicina.

₹ Fue presentada como tesis de bachiller para obtener el título de Médico Cirujano por Liendo GR., Castro CL. Cansancio y somnolencia en conductores de ómnibus interprovinciales con base en Lima, según condiciones laborales de la empresa de transportes [Tesis de Bachiller]. Lima: Facultad de Medicina, Universidad Peruana Cayetano Heredia; 2009.
}

Recibido: 13-04-10 Aprobado: 02-06-10 


\section{INTRODUCCIÓN}

El sueño, es un estado reversible, de respuesta disminuida a estímulos y poco movimiento para el cual usualmente adoptamos una postura determinada ${ }^{(1)}$, siendo un fenómeno biológico activo, cíclico y necesario para la supervivencia ${ }^{(2)}$. Se han planteado diversas hipótesis acerca de su función como son la restauración y recuperación de procesos bioquímicos y fisiológicos inherentes al organismo y la conservación de energía para que el individuo sea capaz de mantener ecuanimidad tanto física como intelectual ${ }^{(1)}$. En contraposición, la privación o falta de sueño deteriora variables funcionales psicomotoras y neurocognitivas, tales como el tiempo de reacción, la capacidad de vigilancia, juicio y atención, así como el procesamiento de la información (3); factores determinantes de un adecuado desempeño laboral. La somnolencia diurna excesiva causa además problemas de concentración, memoria y estados de humor que tienen impacto negativo en el desempeño académico y laboral (4).

Existen actividades que demandan del trabajador todas las capacidades mencionadas, de lo contrario podrían estar atentando contra su vida y la de terceros ${ }^{(2,3,5-19)}$. Tal es el caso de la profesión médica, donde se ha documentado que existe asociación entre los accidentes de tránsito y el trabajar turnos u horas extra en internos de medicina ${ }^{(5)}$.

Es muy importante asegurar un adecuado estado de vigilia en conductores de vehículos de transporte y, con mayor razón, en ómnibus que realizan servicio interprovincial, dado que el trabajo de estos transportistas es muchas veces monótono, durante períodos prolongados y sujeto a cambios de horarios diurnos y nocturnos. Esta actividad laboral es particularmente riesgosa, especialmente $\mathrm{si}$ los conductores duermen pocas horas ${ }^{(2-21)}$.

Diversos trabajos de investigación, tanto nacionales como extranjeros, han descrito la relación directa entre accidentes de tránsito y somnolencia de los conductores ${ }^{(2,3,5,7-20)}$ por lo que es imperativo evaluar las causas de somnolencia en dicha población. Rey de Castro et al. en un estudio transversal basado en una encuesta prevalidada realizada en 238 conductores de ómnibus informales que circulan por la carretera Panamericana Norte del Perú, encontraron que 56\% tenían cansancio durante la conducción y $65 \%$ lo experimentó durante horas de la madrugada, donde el $32 \%$ reconoció "pestañeo" durante la conducción ${ }^{(3)}$. EI mismo autor describió en una revisión periodística del diario "El Comercio", que entre los años 1999-2000 en el Perú; $48 \%$ de los accidentes de tránsito publicados en ese diario podrían haber estado relacionados con la somnolencia durante la conducción ${ }^{(6)}$.
En nuestro medio y en el extranjero, no existen estudios comparativos entre poblaciones de conductores formales e informales de ómnibus interprovinciales. La mayoría de investigaciones se han implementado en terminales informales. Además, considerando que los propietarios de empresas de transporte formal siempre tratan de establecer distancias y remarcar diferencias con las empresas informales, pretendemos con este estudio comparar ambos grupos en materia de cansancio y somnolencia entre sus conductores.

\section{MATERIALES Y MÉTODOS}

\section{TIPO DE ESTUDIO}

Estudio transversal comparativo.

\section{POBLACIÓN Y MUESTRA}

Dado que no se tiene información sobre el total de conductores de ómnibus interprovinciales en nuestro país, calculamos el tamaño muestral basándonos en resultados de un estudio previo realizado en un terminal de Lima (3); considerando al $56 \%$ como frecuencia máxima esperada de somnolencia. Para un estudio descriptivo, siendo el $\alpha=0,05$, el tamaño muestral obtenido con el programa STATA versión 9.0 fue 305. Para la realización de la encuesta, se visitaron terminales formales e informales, talleres y estaciones de servicio de buses interprovinciales en la ciudad de Lima durante el mes de enero de 2009.

Se encuestó a 373 conductores de ómnibus interprovinciales, de los cuales 28 se negaron a participar en el estudio, obteniéndose una tasa de rechazo del $7,5 \%$. Participaron 345 conductores de ómnibus interprovinciales pertenecientes a 100 empresas de transporte público, con base de operaciones en Lima Metropolitana. De acuerdo con la calificación del Ministerio de Transportes y Comunicaciones (MTC), 17 empresas estaban registradas como formales ${ }^{(22)}$.

\section{CLASIFICACIÓN}

Se clasificó a los conductores en Formales e Informales mediante la aplicación de una Ficha de Formalidad pautada arbitrariamente por nosotros, basándonos en el cumplimiento de las principales normas del Reglamento Nacional de Administración de Transportes, Tránsito y Vehículos del MTC (23-25).

Criterios de calificación de formalidad en conductores encuestados:

- Cumplimiento de horarios de salida y llegada de los buses según la programación de la empresa. 
- Disponer de más de dos conductores (para realizar turnos) en recorridos de más de $400 \mathrm{~km}$.

- Número total de horas de conducción no mayor de 10 horas en un periodo de 24 horas.

- Conductores con periodos de vacaciones remunerados no menores de 15 días en un lapso de doce meses.

- Los conductores no duermen o descansan en las bodegas del ómnibus durante el recorrido de la unidad o cuando se encuentra estacionada en el garaje de la empresa o terminal terrestre.

Se consideró formal al conductor que cumple los cinco criterios descritos, de lo contrario fue catalogado como informal.

\section{INSTRUMENTO Y DEFINICIÓN DE TÉRMINOS}

Se utilizó un cuestionario de dos secciones. La primera fue un cuestionario dirigido de 34 preguntas (13 ítems cerrados, 15 abiertos y 6 semiabiertos de tipo cualitativo y cuantitativo), basado en una encuesta aplicada en conductores del cono norte de Lima, Perú (3); los autores responsables del diseño de dicha encuesta autorizaron su empleo en nuestro estudio. En la segunda parte se aplicó la Escala de Somnolencia de Epworth (ESS) ${ }^{(26)}$, adaptada al español por Ferrer et al. (27) y validada por Chiner et al. (28). Dicha escala fue modificada en el Perú (29), agregando situaciones más acordes con la realidad de nuestro país en los ítems número 3 y 4 . Un puntaje igual o mayor a 10 se considerará positivo para la variable somnolencia. No se realizó un proceso de validación para la modificación de la escala antes mencionada.
La variable cansancio fue medida de manera subjetiva, para lo cual se utilizó una escala de Lickert: se preguntó a cada conductor si nunca, raras veces, algunas veces, muchas veces o siempre había sentido cansancio durante la conducción considerándose como positivas desde raras veces hasta siempre.

\section{ANÁLISIS DE DATOS}

Las respuestas obtenidas en las encuestas fueron codificadas y posteriormente ingresadas a una base de datos en Microsoft Excel $®$ Versión 2003. El análisis estadístico se realizó aplicando el paquete Epi Info ${ }^{\mathrm{TM}}$ Versión 3.5.1 (CDC, AT, USA, 2008). En las variables numéricas se emplearon medidas de tendencia central y de dispersión, media aritmética y desviación estándar, respectivamente. En las variables categóricas se usó medidas de frecuencia absoluta y relativa (porcentaje).

Para establecer asociación entre variables categóricas usamos la prueba Ji cuadrado o prueba exacta de Fisher, según corresponda. En el caso de la comparación entre el grupo de formales e informales para variables numéricas se usó la prueba T de Student para grupos independientes previa comprobación de los supuestos de normalidad y de homogeneidad de varianzas. A pesar de no contar con un muestreo probabilístico se asumió como significativo cuando el valor $p$ fue menor de 0,05.

\section{ASPECTOS ÉTICOS}

La participación de los encuestados fue voluntaria y anónima. El estudio fue revisado y aprobado por el Comité Institucional de Ética de la Universidad Peruana Cayetano Heredia (Código SIDISI: 0000053024).

Tabla 1. Características observadas entre conductores formales e informales.

\begin{tabular}{|c|c|c|c|}
\hline & $\begin{array}{c}\text { Formales }(\mathrm{N}=71) \\
\mathrm{x} \pm \mathrm{ds}\end{array}$ & $\begin{array}{c}\text { Informales }(\mathrm{N}=\mathbf{2 7 4}) \\
\mathrm{x} \pm \mathrm{ds}\end{array}$ & $\mathbf{p}$ \\
\hline Edad (años) & $44,45 \pm 9,08$ & $44,41 \pm 9,52$ & $0,51^{*}$ \\
\hline Índice de Masa Corporal (IMC) & $28,38 \pm 3,44$ & $28,19 \pm 3,77$ & $0,66^{*}$ \\
\hline Tiempo de trabajo como chofer (años) & $16,28 \pm 8,60$ & $17,05 \pm 9,63$ & $0,74^{*}$ \\
\hline Tiempo de trabajo en la empresa (años) & $5,41 \pm 6,05$ & $4,40 \pm 4,79$ & $0,90^{*}$ \\
\hline \multirow[t]{2}{*}{ Horas de sueño en 24 horas } & $6,87 \pm 2,03$ & $6,94 \pm 1,80$ & $0,77^{*}$ \\
\hline & n (\%) & n (\%) & \\
\hline \multicolumn{4}{|l|}{ Grado de Instrucción } \\
\hline Primaria & $1(1,4)$ & $15(5,5)$ & $0,34^{* *}$ \\
\hline Secundaria & $60(84,5)$ & $220(80,3)$ & \\
\hline Técnico o superior & $10(14,1)$ & $39(14,2)$ & \\
\hline Sin seguro médico & $7(9,9)$ & $89(32,5)$ & $0,11^{* *}$ \\
\hline Obesidad (IMC>30) & $21(29,6)$ & $74(27,0)$ & $0,42^{* *}$ \\
\hline Somnolencia \# & $18(25,4)$ & $38(14,0)$ & $0,47^{* *}$ \\
\hline
\end{tabular}

$\mathrm{x} \pm$ ds: media \pm desviación estándar, \# definida como un valor mayor de 10 en la escala de Epworth (ESE), * T de Student, ** Ji cuadrado. 


\section{RESULTADOS}

\section{CARACTERÍSTICAS GENERALES}

Se implementó un total de 345 encuestas y, de acuerdo con los criterios señalados, 71 fueron conductores formales y 274 informales. Del total de participantes, 134 pertenecían a 17 empresas consideradas como "formales" por el MTC. De acuerdo con nuestros criterios, sólo $32 \%$ de ellos eran formales. Todos los participantes fueron varones, con edades que fluctuaron entre 23 y 70 años. En la Tabla 1 se resumen las características de la población encuestada.

\section{ASPECTOS RELACIONADOS CON LA CONDUCCIÓN, CANSANCIO, SOMNOLENCIA Y CONDICIONES LABORALES}

En un periodo de 24 horas, 34 de 71 (48\%) conductores formales y 118 de 274 (43\%) informales, duermen menos de siete horas, no existiendo diferencias estadísticamente significativas entre ambos grupos. La información relacionada con el número de horas de sueño durante la noche de conductores diurnos

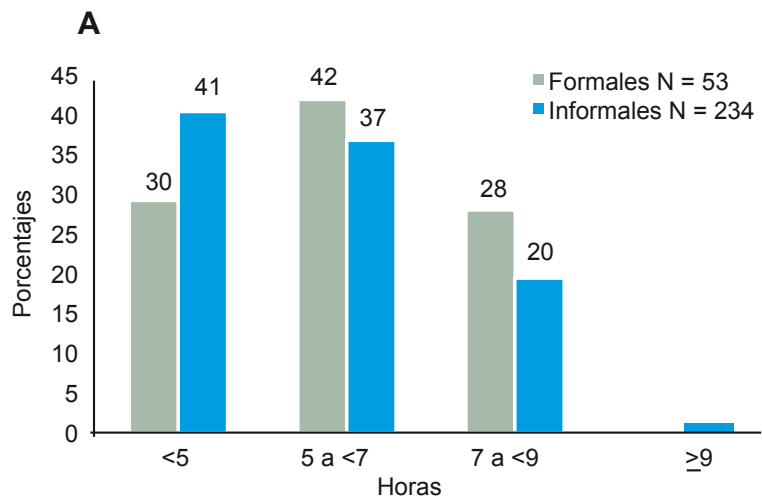

B

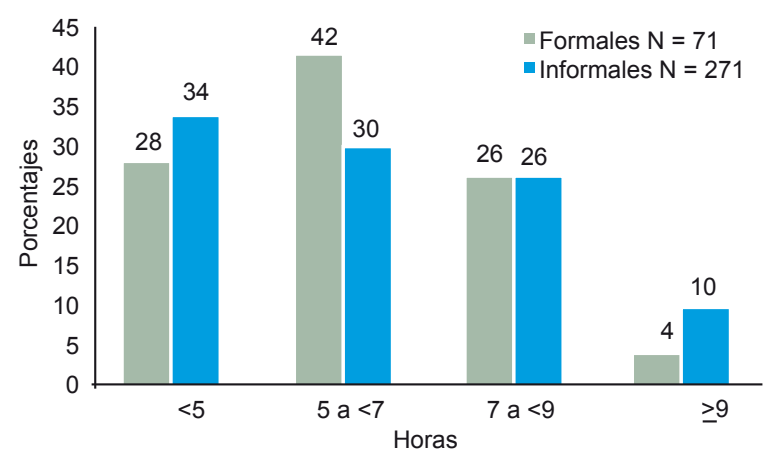

Figura 1. Horas de sueño en conductores formales e informales. A. Choferes que trabajan turno diurno; B. Choferes que trabajan turno nocturno. y el número de horas de sueño durante el día, de conductores nocturnos se presenta en la Figura $1 \mathrm{~A} y$ $1 \mathrm{~B}$, respectivamente.

De la misma manera, en un periodo de 24 horas, $71(26 \%)$ choferes informales manejaron más de 9 horas y $42(15 \%)$ más de 12 horas. Asimismo, 54 (76\%) conductores formales y 171 (62\%) conductores Informales conducen más de cuatro horas continuas.

Por definición, un conductor formal no debe dormir en la bodega del ómnibus; el número, porcentaje y distribución de conductores informales que duermen en la bodega o maletero se presenta en la Figura 2. Cuando el ómnibus se encuentra en el terminal terrestre, 48 (67\%) formales y 120 (42\%) informales duermen en una habitación especial designada para ése propósito, mientras que $13(18 \%)$ y 96 (34\%) formales e informales, respectivamente, lo hacen en el ómnibus.

Treinta y nueve (55\%) choferes formales y 159 (58\%) informales refirieron roncar y 17 (24\%) formales y 69 $(25 \%)$ informales mencionaron que tenían pausas respiratorias. Asimismo, 48 (68\%) choferes Formales presentaron cansancio frente a 192 (70\%) informales $(p=0,37)$. Diez $(14 \%)$ choferes formales y $69(25 \%)$ informales tuvieron pestañeo durante la conducción.

Las principales maniobras que los conductores usualmente emplean para evitar quedarse dormidos mientras conducen se presentan en la Tabla 2; donde el cambio de conductor fue la más frecuente tanto en conductores formales como informales.

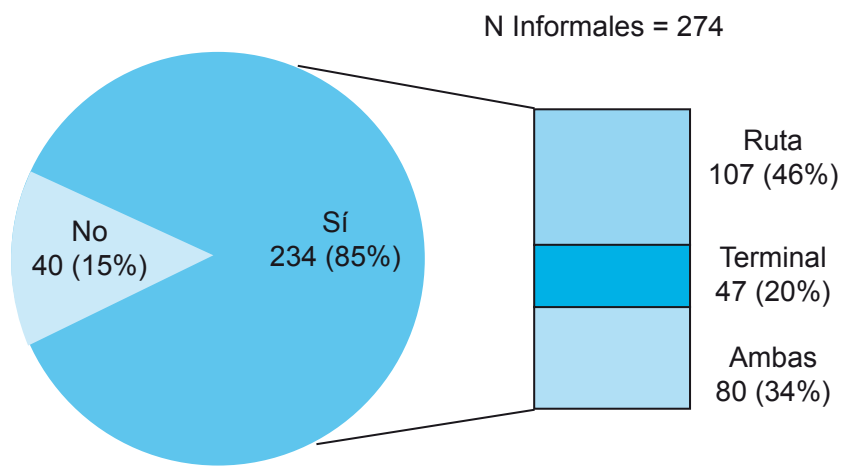

Figura 2. Choferes informales que duermen en la bodega del omnibús: frecuencia y lugar donde lo hacen. 
Tabla 2. Maniobras usadas por los conductores para evitar quedarse dormidos *.

\begin{tabular}{lcc}
\hline & $\begin{array}{c}\text { Formales } \\
\mathbf{( N = 7 1 )} \\
\mathbf{N}(\%)\end{array}$ & $\begin{array}{c}\text { Informales } \\
\mathbf{( N = 2 7 4 )} \\
\mathbf{N}(\%)\end{array}$ \\
\hline Cambio de conductor & $24(33,8)$ & $153(55,8)$ \\
Escuchar música & $22(31,0)$ & $76(27,7)$ \\
\hline Comer fruta & $16(22,5)$ & $54(19,7)$ \\
\hline Abrir la ventana del vehículo & $10(14,1)$ & $40(14,6)$ \\
\hline Mojarse la cara & $8(11,3)$ & $60(21,9)$ \\
\hline Tomar café & $8(11,3)$ & $22(8,0)$ \\
\hline Beber gaseosas & $7(9,9)$ & $27(9,9)$ \\
agua mineral & $7(9,9)$ & $22(8,0)$ \\
\hline Fumar & $6(8,5)$ & $13(4,7)$ \\
Comer snacks/ masticar chicle & $5(7,0)$ & $11(4,0)$ \\
Reducir la velocidad & $1(1,4)$ & $8(2,9)$ \\
Chacchar coca & $1(1,4)$ & $4(1,5)$ \\
Curado (café + pastillas) & $1(1,4)$ & $0(0,0)$ \\
Bebidas energizantes & $1(1,4)$ & $4(1,5)$ \\
Conversar & $1(1,4)$ & $1(0,4)$ \\
Estacionar el ómnibus y & $0(0,0)$ & $5(1,8)$ \\
caminar/ bajar del ómnibus & $0(0,0)$ & $5(1,8)$ \\
\hline Otros & &
\end{tabular}

* Los conductores pueden reportar más de una mabiobra.

En cuanto a accidentes de tránsito, es importante mencionar que 34 conductores formales (48\%) y 135 informales $(49 \%)$ tuvieron un accidente o estuvieron a punto de tenerlo. Se preguntó por la hora en que se produjo el accidente o casi accidente y los resultados se presentan en la Figura 3.

La madrugada es el momento del día en el que la población encuestada sintió más cansancio: 35 (70\%) formales y 126 (62\%) informales. Asimismo, 71 $(100 \%)$ conductores formales y $271(99 \%)$ conductores informales manejaban durante la noche. El número de

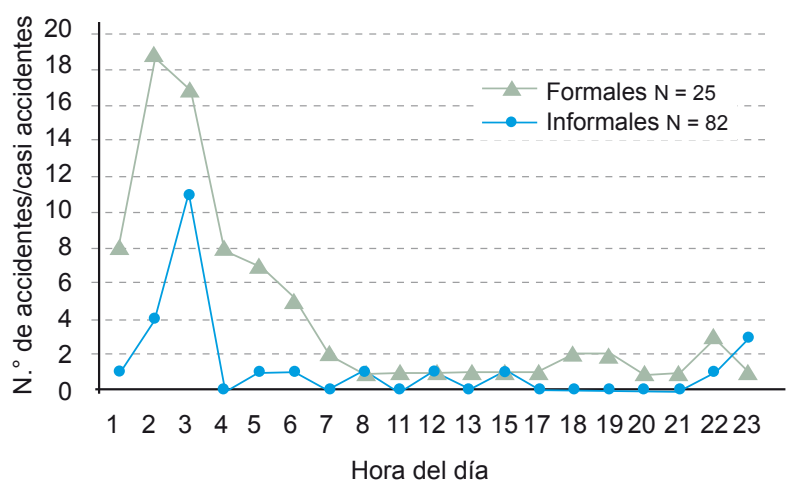

Figura 3. Horas de ocurrencia de accidente o casi accidentes de choferes formales e informales.

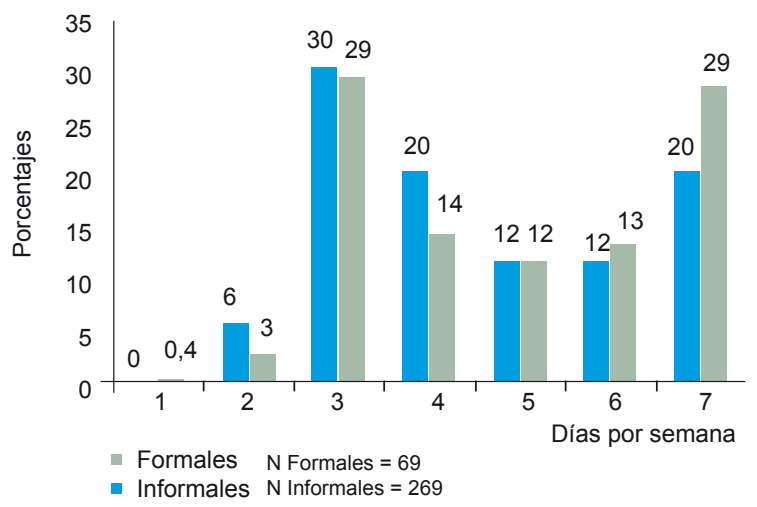

Figura 4. Días de turno nocturno por semana en choferes formales e informales.

días de conducción nocturna a la semana, para ambos grupos se presenta en la Figura 4.

Se indagó por la causa, atribuida por el conductor, de su accidente o casi accidente encontrándose que el error de otro conductor, $18(47 \%)$ formales y $80(53 \%)$ informales, fue la más frecuente; luego el cansancio, 5 (13\%) formales y $23(15 \%)$ informales; posteriormente, carretera en mal estado, 4 (11\%) formales y $12(8 \%)$ informales; seguido por, falla mecánica, $4(11 \%)$ formales y $7(5 \%)$ informales; entre otros. La negligencia y descuido del conductor, así como, el cansancio fueron consideradas como las principales causas de accidentes de carreteras por 29 $(32 \%)$ de los formales y $106(30 \%)$ de los informales y por $26(28 \%)$ formales y $84(24 \%)$ informales; respectivamente. Finalmente, $190(55 \%)$ conductores, conocían algún chofer que se había accidentado por cansancio.

Del total de la muestra, 56 (16\%) conductores tuvieron un puntaje mayor a 10 en la Escala de Somnolencia de Epworth. Presentaron Somnolencia: 18 (25\%) conductores Formales y $38 \quad(14 \%)$ conductores Informales. No se encontró diferencia estadísticamente significativa entre ambos grupos.

De los encuestados que presentaron valor positivo para somnolencia de acuerdo al puntaje de ESE, 6 (18\%) conductores Formales y 15 (11\%) Informales tuvieron antecedente de accidente $o$ casi accidente $(p=0,34)$.

\section{DISCUSIÓN}

El principal medio de transporte empleado en nuestro país y en la mayor parte del mundo, es el terrestre; por razones de mayor demanda debido al costo, accesibilidad, logística, entre otras. Por ello consideramos importante evaluar aspectos relacionados con los servicios de transporte interprovincial empleados por la alta proporción de usuarios en nuestro país. 
En lo que respecta a cantidad de horas de sueño empleadas por los conductores durante un día, Rey de Castro et al. en un estudio realizado en el terminal terrestre informal más grande de Lima Metropolitana, evidenciaron que $47 \%$ de los conductores duermen menos de siete horas al día y $40 \%$ menos de seis ${ }^{(3)}$.

Asimismo, en un estudio realizado en Carolina del Norte por Sttuts et al. demostraron que $40 \%$ de conductores de vehículos duermen menos de siete horas al día ${ }^{(7)}$. La media de horas de sueño descritas en conductores de ómnibus en Argentina fue 3,76 durante el día laboral ${ }^{(8)}$. Los valores mencionados son similares a los encontrados en nuestro estudio. Esta información revela que en muestras de poblaciones distintas de conductores de vehículos, la tendencia de esta variable es similar, incluyendo grupos de conductores formales e informales.

La gran mayoría de choferes de ómnibus interprovinciales conducen seis o más horas al día y lo que llamó la atención es que las dos terceras partes de los conductores formales y más de la mitad de informales lo hacen en periodos mayores de cuatro horas continuas, violando las recomendaciones del Ministerio de Transportes y Comunicaciones estipuladas en el Reglamento Nacional de Administración de Transportes, que limitan el periodo de conducción continua a cuatro horas ${ }^{(23)}$. Este hallazgo fue descrito anteriormente en una publicación nacional, por un estudio realizado en conductores informales, encontrándose que cerca de $50 \%$ de conductores manejan más de cinco horas seguidas ${ }^{(3)}$. Nuestro estudio confirma esta peligrosa violación normativa en la muestra evaluada.

Pérez Chada et al., encontraron en Argentina que la media de horas de conducción por día en choferes de camiones de carga fue 15,9 ${ }^{(8)}$. Leechawengwongs $M$. et al. señalan que $61 \%$ de conductores de ómnibus o camiones comerciales tailandeses conducían cerca de 12 horas diarias ${ }^{(9)}$. En nuestro estudio 15\% de conductores informales trabajó más de 12 horas al día. Si bien es cierto este valor es menor a lo encontrado en otros países, no deja de ser relevante ya que existe asociación entre cantidad de horas de conducción y somnolencia durante la conducción.

Una de las expresiones más notorias relacionadas con los hábitos del sueño de los conductores, es la relacionada con el lugar donde éstos duermen, estando en el terminal o en ruta y cuando su compañero le reemplaza al volante. Dormir en la bodega del vehículo es una costumbre peligrosa ya que no está diseñada para dicho propósito, no tiene adecuada ventilación, la puerta de acceso sólo se abre desde el exterior y, finalmente, el espacio es reducido lo que resulta muy incómodo para lograr un buen sueño. De acuerdo con la definición empleada por nosotros,
$85 \%$ de los informales empleaban esta costumbre, cifra parecida a encontrada en el Terminal Fiori (80\%) ${ }^{(3)}$ y más alta que la descrita en el estudio realizado en el Terminal de Huancayo, de $63 \%{ }^{(20)}$. De acuerdo con estos hallazgos, la costumbre de dormir en la bodega es muy alta en conductores informales por lo que la normativa específica no viene cumpliéndose.

En la presente investigación; más de la mitad de conductores, en ambos grupos, roncan al dormir y casi la cuarta parte de formales e informales tienen pausas respiratorias durante el sueño. Ambos hallazgos son parte de los síntomas cardinales del síndrome de apnea-hipopnea del sueño (SAHS) causa orgánica de somnolencia asociada con el riesgo de accidentes durante la conducción ${ }^{(30)}$.

El cansancio que pueda o no sentir un conductor de ómnibus interprovincial, es consecuencia de la interacción de varios factores: horas de sueño, horas de trabajo, hábitos de sueño, enfermedades orgánicas como el SAHS o la narcolepsia, entre otros. Hemos constatado que la presencia de cansancio en ambos grupos fue elevada y con cifras similares. Los porcentajes de conductores que pestañean durante la conducción son altos.

Los accidentes de tránsito son muy comunes entre conductores de ómnibus interprovinciales y sus consecuencias en la salud pública de un país es enorme; asimismo, el impacto económico que representan los trastornos del sueño y las repercusiones de estos para un país, es considerable ${ }^{(21,31)}$.

En Australia durante el año 2004, el costo total producto de desórdenes del sueño fue de \$ 7494 millones que incluyó \$808 millones para cubrir exclusivamente gastos relacionados al rubro salud debido a accidentes de tránsito consecuencia de la somnolencia durante la conducción ${ }^{(31)}$.

En el presente estudio encontramos que la frecuencia de accidentes o casi accidentes se concentró en horas de la madrugada, siendo el comportamiento de ambos grupos bastante similar. Rey de Castro et al. y Rosales et al. reportan frecuencias parecidas, de esta variable, en sus respectivos estudios ${ }^{(3,20)}$. Wylie et al. describen que la hora de conducción y sobre todo la madrugada, tiene mayor influencia en el cansancio del conductor que, incluso, el tiempo de conducción y el número de horas de viajes acumulados ${ }^{(10)}$.

De acuerdo con esta investigación más de la mitad de conductores tienen menos horas de sueño en el periodo de 24 horas, sienten mayor cansancio o somnolencia durante las madrugadas, el $100 \%$ conducen de noche 
y más de la mitad lo hacen en ese turno más de cuatro veces por semana.

Para ambos grupos de conductores las dos principales causas de accidentes de tránsito son negligencia, imprudencia o descuido del conductor y cansancio respectivamente; sin embargo, el porcentaje atribuido a cansancio aumenta cuando el entrevistado habla de terceras personas. Cabe mencionar que en un estudio previo en el terminal de Fiori, la variable "cansancio" fue la respuesta más frecuente $(55 \%)$ como causa de accidentes seguida de imprudencia del chofer $24 \%{ }^{(3)}$. Rosales et al., documentaron que $82 \%$ de conductores atribuían al cansancio como causa del accidente o casi accidente ${ }^{(20)}$.

Un porcentaje considerable de encuestados presentó somnolencia, de acuerdo con la ESS, sin diferencias entre ambos grupos. En el estudio realizado en Argentina por Pérez Chada et al., se evidenció que $80 \%$ de conductores tenía privación de sueño y de esta población $14 \%$ obtuvo un puntaje mayor a 10 en la ESE ${ }^{(16)}$; resultados similares a la presente investigación. En tal sentido, 16\% de somnolencia en el total de la muestra se considera un porcentaje alto debido a la peligrosidad que implica un conductor somnoliento que lleva en su unidad 30 o 40 pasajeros.

Por el diseño y las características del estudio presentado, existen algunas limitaciones, como la realización de un muestreo no probabilístico, lo que constituye un sesgo de selección; además, un cálculo de tamaño muestra para un estudio de prevalencia sin considerar la potencia estadística para efectos de análisis comparativo. Asimismo, la no validación de la modificación de la Escala de Somnolencia de Epworth, resulta en un sesgo de medición; agregado a ello, la posible omisión de información por parte de los encuestados, que establece un sesgo de información; y, finalmente, la confusión entre los términos "cansancio" y "somnolencia", pueden ser factores limitantes en el estudio. No obstante, esto último ha sido planteado anteriormente por investigadores nacionales, donde se pudo identificar la connotación de estos términos en la población de estudio. Los conductores utilizaron la palabra "cansancio" para expresar sensación de falta de energía, pesadez de cabeza o cuello, modorra, disminución de la capacidad de atención o concentración y somnolencia indistintamente. En cambio, respondieron invariablemente en forma negativa al ser interrogados por "somnolencia" o "quedarse dormido" durante la conducción (11).

El comportamiento de los grupos formal e informal es similar. Los hábitos de sueño y de manejo son parecidos y las consecuencias de la privación de sueño tienen frecuencias semejantes. Las normativas no aparentan ser efectivas y el control por las autoridades correspondientes es, a todas luces, deficiente.
Como premisa, la clasificación de formalidad en nuestro estudio ha sido dirigida a los conductores y no a las empresas de transporte. Documentamos objetivamente que dentro de empresas clasificadas como formales por el MTC, un porcentaje considerable de conductores no respetan pautas mínimas de formalidad fijadas por el organismo regulador de transporte en el país. En la muestra que aquí se presenta, las empresas que afirman ser formales tienen claro perfil de informalidad.

Es primordial asegurar que todos los trabajadores tengan las mismas condiciones laborales, proveer espacios especialmente acondicionados para que los conductores descansen después de las jornadas de trabajo y limitar horarios de conducción prolongados. Se debe fomentar campañas informativas y fortalecer la aplicación de normas existentes, haciendo hincapié en los factores de riesgo para cansancio y somnolencia.

De acuerdo con estos hallazgos: es imperativo que choferes y propietarios de las empresas conozcan los riesgos de la trabajar en horario nocturno, tomen las medidas necesarias para disminuir el riesgo de accidentes, especialmente en las madrugadas. Ello devendría concretamente en disminuir la oferta de turnos nocturnos a los usuarios.

La información que se puede obtener en conductores del sector público es importante, sobre todo si se emplea el método de la investigación científica con un instrumento tan sencillo como un cuestionario bien diseñado y validado. Ello permite identificar problemas específicos y formular planes de intervención que beneficien al conductor, usuario, transportista y al país.

Es aconsejable diseñar nuevos estudios con objetivos similares, en conductores de camiones y colectivos interprovinciales así como en el sistema de transporte público de Lima Metropolitana.

\section{Fuente de Financiamiento}

Autofinanciado.

\section{Conflictos de Interés}

Los autores declaran no tener conflictos de interés en la publicación de este artículo.

\section{REFERENCIAS BIBLIOGRÁFICAS}

1. World Federation of Sleep Research Societies. Sleep Syllabus: basics of sleep behavior. What is sleep? [Página Web]. WebSciences International.; 1998. [Fecha de acceso: 31 de enero de 2009] Disponible en: http://www. sleephomepages.org/sleepsyllabus/a.html 
2. Souza J, Paiva T, Reimao R. Sleep habits, sleepiness and accidents among truck drivers. Arq Neuropsiquiatr. 2005; 63(4): 925-30.

3. Rey de Castro J, Gallo J, Loureiro H. Cansancio y somnolencia en conductores de ómnibus y accidentes de carretera en el Perú: estudio cuantitativo. Rev Panam Salud Publica. 2004; 16(1): 11-18.

4. Guilleminault $\mathbf{C}$, Brooks S. Excessive daytime sleepiness: a challenge for the practising neurologist. Brain. 2001; 124(pt 8): 1482-91.

5. Barger LK, Cade BE, Ayas NT, Cronin JW, Rosner B, Speizer FE, et al. Extended work shifts and the risk of motor vehicle crashes among interns. N Engl J Med. 2005; 352(2): 125-34.

6. Rey de Castro J. Accidentes de tránsito en carreteras e hipersomnia durante la conducción. ¿Es frecuente en nuestro medio? La evidencia periodística. Rev Med Hered. 2003; 14(2): 69-73.

7. Sttuts J, Wilkins J, Osberg J, Vaughn B. Driver risk factors for sleep-related crashes. Accid Anal Prev. 2003; 35(3): 321-31.

8. Pérez-Chada D, Videla AJ, O'Flaherty ME, Palermo $\mathbf{P}$, Meoni J, Sarchi MI, et al. Sleep habits and accident risk among truck drivers: a cross-sectional study in Argentina. Sleep. 2005; 28(9): 1103-8.

9. Leechawengwongs $M$, Leechawengwongs $E$, Sukying C, Udomsubpayakul U. Role of drowsy driving in traffic accidents: a questionnaire survey of Thai commercial bus/ truck drivers. J Med Assoc Thai. 2006; 89(11): 1845-50.

10. Leger $\mathbf{D}$. The cost of sleep-related accidents: a report for the National Commission on Sleep Disorders Research. Sleep. 1994; 17(1): 84-93.

11. Wylie CD, Schultz T, Miller JC, Mitler MM, Mackie RR. Commercial Motor Vehicle Driver Fatigue and Alertness Study: Technical Summary (TP12876E) . Washington DC: Federal Highway Administration; 1996.

12. Rey de Castro J, Soriano S. Hipersomnia durante la conducción de vehículos ¿causa de accidentes en carreteras? A propósito de un estudio cualitativo. Rev Soc Peru Med Interna. 2002; 15:142-9.

13. Masa JF, Rubio M, Findley LJ. Habitually sleepy drivers have a high frequency of automobile crashes associated with respiratory disorders during sleep. Am J Respir Crit Care Med. 2000; 162 (4Pt 1): 1407-12.

14. Lyznicki JM, Doege TC, Davis RM, Williams MA. Sleepiness, driving, and motor vehicle crashes. JAMA. 1998; 279(23): 1908-13.

15. Philip P, Akerstedt T. Transport and industrial safety, how are they affected by sleepiness and sleep restriction? Sleep Med Rev. 2006; 10(5): 347-56.

16. De Pinho RS, Da Silva-Junior FP, Bastos JP, Maia WS, De Mello MT, De Bruin VM, et al. Hypersomnolence and accidents in truck drivers: a cross-sectional study. Chronobiol Int. 2006; 23(5): 963-71.

17. Santos EH, de Mello MT, Pradella-Hallinan M, Luchesi L, Pires ML, Tufik S. Sleep and sleepiness among Brazilian shift-working bus drivers. Chronobiol Int. 2004; 21(6): 881-8.

18. Mello MT, Santana MG, Souza LM, Oliveira PC, Ventura ML, Stampi C, et al. Sleep patterns and sleep-related complaints of Brazilian interstate bus drivers. Braz J Med Biol Res. 2000; 33(1):71-7.
19. Carter N, Ulfberg J, Nyström B, Edling C. Sleep debt, sleepiness and accidents among males in the genera population and male professional drivers. Accid Anal Prev. 2003; 35(4): 613-17.

20. Rosales E, Egoavil M, Durand I, Montes N, Flores R, Rivera S, et al. Accidentes de carretera y su relación con cansancio y somnolencia en conductores de ómnibus. Rev Med Hered. 2009; 20(2): 48-59.

21. Leger $\mathbf{D}$. The cost of sleep-related accidents: a report for the National Commission on Sleep Disorders Research. Sleep. 1994; 17(1): 84-93.

22. Ministerio de Transportes y Comunicaciones. Ranking de las Empresas de Transporte Interprovincial Terrestre de Pasajeros, según flota, concesiones y frecuencias. [Documento en Internet]. Lima: Ministerio de Transportes y Comunicaciones; 2007 [Fecha de acceso: 26 de octubre de 2009]; Disponible en: http://www.mtc. gob.pe/estadisticas/ARCHIVOS/ESTADISTICA\%20 items/1-TRANSPORTES/4.CARRETERO/SERVICIOS/ PASAJEROS/3.A.14.xls

23. Perú, Ministerio de Transportes y Comunicaciones. Reglamento nacional de administración de transportes. Decreto Supremo $N^{\circ}$ 040-2001-MTC de 28 de julio de 2001. Lima: MTC; 2001.

24. Perú, Ministerio de Transportes y Comunicaciones. Reglamento nacional de tránsito. Decreto Supremo 033 2001-MTC de 24 de julio del 2001. Lima: MTC; 2001.

25. Perú, Ministerio de Transportes y Comunicaciones. Reglamento nacional de vehículos. Decreto Supremo 058 2003-MTC de 07 de octubre del 2003. Lima: MTC; 2003.

26. Johns MW. A new method for measuring daytime sleepiness: the Epworth sleepiness scale. Sleep. 1991; 14(6): 540-45.

27. Ferrer M, Vilagut G, Monasterio C, Montserrat JM Mayos M, Alonso J. Medida del impacto de los trastornos del sueño: las versiones españolas del cuestionario del impacto funcional del sueño y de la Escala de Somnolencia de Epworth. Med Clin (Barc). 1999; 113(7): 250-55.

28. Chiner E, Arriero JM, Signes-Costa J, Marco J, Fuentes I. Validation of the Spanish version of the Epworth Sleepiness Scale in patients with a sleep apnea syndrome. Arch Bronconeumol. 1999; 35(9): 422-27.

29. Rey de Castro J, Vizcarra D, Alvarez J. Somnolencia diurna y síndrome de apnea hipopnea del sueño. Rev Soc Peru Med Interna. 2003; 16(2): 74-83.

30. Newman AB, Nieto FJ, Guidry U, Lind BK, Redline S Pickering TG, et al. Relation of sleep-disordered breathing to cardiovascular risk factors: the Sleep Heart Health Study. Am J Epidemiol. 2001; 154(1): 50-59.

31. Hillman DR, Murphy AS, Pezzullo L. The economic cost of sleep disorders. Sleep. 2006; 29(3): 282-83.

Correspondencia: Gustavo R. Liendo.

Dirección: Av. Santa Cruz 1254. Dpto 302, Miraflores, Lima 18,

Perú.

Teléfono: (511)-4427333

Correo electrónico: gustavoliendo@yahoo.com 